\title{
Alterações hemogasométricas em equinos submetidos à distensão do cólon menor
}

[Blood gas alterations in horses subjected to small colon distention]

\author{
A.F. Sabes, K. Gravena, M.C. Herdandez-Tovar,V.A. Canello, N.S. Bernardi, \\ D.J. Queiroz, J.A. Oliveira, J.C. Lacerda-Neto \\ Universidade Estadual Paulista "Júlio de Mesquita Filho" - Unesp - Jaboticabal, SP
}

\section{RESUMO}

O objetivo do presente estudo foi avaliar a hemogasometria venosa de equinos com lesões isquêmicas induzidas experimentalmente no cólon menor. Foram utilizados oito equinos sadios, com idades entre cinco e oito anos, sem raça definida. Os animais foram submetidos à celiotomia e a quatro horas de obstrução intraluminal do cólon menor. Foram realizadas coletas de amostras de sangue imediatamente antes da indução anestésica (T0), no momento em que a anestesia foi estabilizada (T1), quatro horas após a obstrução intraluminal (T4), e, durante o pós-cirúrgico, as coletas foram realizadas em intervalos de 12 horas até completar 72 horas (T16, T28, T40, T52, T64 e T76). Notou-se em T4 alcalose metabólica, com compensação respiratória por meio da hipoventilação. Esse quadro de alcalose foi brando e transitório, retornando os valores normais para a espécie em T16, com 12 horas de desobstrução intestinal.

Palavras-chave: cavalo, gases sanguíneos, obstrução

\begin{abstract}
The objective of this study was to evaluate the blood gas analysis of venous blood of horses with experimentally induced ischemic lesions on the lower colon. Eight healthy horses were used, with ages between five and eight years, mixed breed. The animals were subjected to celiotomy and four hours of lower colonic intraluminal obstruction. The harvests were made with the blood samples immediately before induction of anesthesia (TO), when the anesthesia was stabilized (T1), 4 hours after the intraluminal obstruction (T4) and during postsurgical times were performed at intervals of 12 hours to complete 72 hours (T16, T28, T40, T52, T64 and T76). The occurrence of metabolic alkalosis on T4 with respiratory compensation by hypoventilation was noted, this alkalosis period was bland and transient, returning the normal values for the specie on T16, 12 hours after the intestinal obstruction.
\end{abstract}

Keywords: horse, blood gas, obstruction

\section{INTRODUÇÃO}

Por possuir peculiaridades anatômicas em seu aparelho digestório, a espécie equina apresenta predisposição a alterações morfofisiológicas graves, responsáveis por sinais de dores abdominais conhecidas como cólica ou abdômen agudo (Peiró e Mendes, 2004). Dentre os inúmeros tipos de cólica, as obstruções intestinais têm sido apontadas como a principal causa de internação e óbitos em equinos em hospitais veterinários de todo o mundo (Faleiros et al., 2007). Estudo retrospectivo sobre abdômen agudo realizado em condições tropicais

Recebido em 28 de outubro de 2016

Aceito em 3 de dezembro de 2016

E-mail: amanda.festa@hotmail.com destacou que as afecções envolvendo o intestino grosso representavam $19 \%$ das ocorrências, dos quais mais da metade $(51,35 \%)$ foram causadas por obstrução do cólon descendente (Corrêa et al., 2006).

As anormalidades eletrolíticas e ácido-base usualmente não definem o diagnóstico, porém algumas enfermidades são caracterizadas por predizerem a tendência nesses parâmetros (Johnson, 1995). O método mais adequado e eficaz para detecção das alterações no equilíbrio acidobásico dos fluidos orgânicos consiste na hemogasometria, por meio da mensuração dos gases sanguíneos, do $\mathrm{pH}$ e do bicarbonato (Klein 
et al.,1999; Silverman e Birks, 2002; Day, 2002; Gokce et al., 2004). De acordo com a intensidade do distúrbio abdominal, a caracterização dos desequilíbrios metabólicos torna-se vital para o diagnóstico precoce e para o direcionamento da conduta clínica e cirúrgica (Nappert e Johnson, 2001).

Os desequilíbrios hidroeletrolíticos e acidobásico podem ser agravantes em equinos com doença gastrintestinal grave. No animal sadio, o volume e a composição dos fluidos orgânicos são mantidos em estreita faixa de variação. Nas enfermidades do trato digestório, os mecanismos de controle se alteram, sobrevindo desequilíbrios que incluem perda de água e eletrólitos e concomitante distúrbio ácido-base (Marques, 1990).

Um dos sinais mais frequentes da síndrome cólica, em equinos, é a desidratação. A hipovolemia decorrente dessa desidratação induz à baixa perfusão tecidual, resultando em limitado fornecimento de oxigênio aos tecidos e diminuição na excreção de íons $\mathrm{H}^{+}$pelos rins (Alves et al., 2005). A hipóxia tecidual aumenta a biossíntese do ácido láctico originário do metabolismo anaeróbico, liberando-o mais rapidamente do que ele pode ser oxidado ou reconvertido em glicose ou glicogênio pelo fígado (Gosset et al., 1987). Esse evento é um dos principais fatores responsáveis pela ocorrência da acidose metabólica nos equinos desidratados (Nappert e Johnson, 2001). Portanto, o objetivo deste estudo foi determinar as alterações hemogasométricas de equinos com obstrução do cólon menor induzida experimentalmente.

\section{MATERIAL E MÉTODOS}

Foram utilizados oito equinos adultos, quatro machos e quatro fêmeas, sem raça definida, com idade de $11 \pm 2$ anos e pesando $370,5 \pm 17,4 \mathrm{~kg}$, alocados em um único grupo. Antes do início do período experimental, os animais passaram por avaliação clínica e hematológica para confirmação da higidez, utilizando-se apenas os animais sadios. Este estudo recebeu aprovação pelo Comitê de Ética da Faculdade de Ciências Agrárias e Veterinárias - FCAV/Unesp Jaboticabal (Protocolo n ${ }^{\circ}$ 007568-09).

Após jejum alimentar e hídrico de 24 e 12 horas, respectivamente, iniciou-se o procedimento anestésico para realização da celiotomia com administração de cloridrato de detomidina $10 \%$ $(0,015 \mathrm{mg} / \mathrm{kg})$ como medicação pré-anestésica. Após 10 minutos, realizou-se indução anestésica por meio da administração de cetamina $10 \%$ $(2,0 \mathrm{mg} / \mathrm{kg})$, à qual seguiu-se a administração de maleato de midazolam $(0,1 \mathrm{mg} / \mathrm{kg})$, levando o animal ao decúbito lateral esquerdo. Ato contínuo, realizou-se intubação orotraqueal e o animal foi posicionado em decúbito dorsal, sobre a mesa cirúrgica. A anestesia inalatória foi mantida com isoflurano vaporizado em circuito semifechado, sob ventilação controlada. Para manutenção da pressão arterial média (PAM), foi utilizado $0,002 \mathrm{mg} / \mathrm{kg} / \mathrm{min}$ de dobutamina, durante todo o procedimento cirúrgico. Também foram administrados $5 \%$ do peso corpóreo de solução de ringer com lactato por dia (IV) para a manutenção da volemia.

O procedimento cirúrgico foi realizado conforme preconizou Gravena et al. (2016). No período pós-cirúrgico imediato, os animais receberam ceftiofur sódico $(2,2 \mathrm{mg} / \mathrm{kg}$ a cada 12 horas), cloridrato de tramadol $(1,1 \mathrm{mg} / \mathrm{kg}$ a cada seis horas) e butilbrometo de escopolamina $(0,2 \mathrm{mg} / \mathrm{kg}$ a cada seis horas) durante três dias consecutivos após celiotomia. Curativos na ferida cirúrgica foram realizados a cada 12 horas com polivinilpirrolidona-iodo tópico a $1 \%$. Durante todo o pós-cirúrgico, os animais foram alimentados apenas com capim fresco, feno de Tifton e água à vontade.

As análises hemogasométricas foram realizadas antes da indução anestésica (T0), na estabilização anestésica (T1), quatro horas após obstrução (T4) e no pós-cirúrgico, em intervalos de 12 horas, completando 72 horas (T16, T28, T40, T52, T64 e T76). O sangue venoso, em todos os momentos, foi obtido por venipunção jugular, utilizando-se seringa própria para avaliação hemogasométrica $(1 \mathrm{ml} / \mathrm{cc}$ insulina U100. Injex $\AA$, Brasil). As leituras do material coletado foram realizadas em analisador de gases, eletrólitos e hemoglobina total (i-STAT analyzer - Abbott Laboratories, Princeton, USA). Determinou-se pressão parcial de oxigênio $\left(\mathrm{PO}_{2}\right)$, pressão parcial de dióxido de carbono $\left(\mathrm{PCO}_{2}\right)$, excesso/déficit de base $(\mathrm{BE})$, saturação de oxigênio $\left(\mathrm{SO}_{2}\right)$, logaritmo negativo da atividade de íons hidrogênio $(\mathrm{pH})$, concentrações de bicarbonato $\left(\mathrm{HCO}_{3}{ }^{-}\right)$, íon sódio $\left(\mathrm{Na}^{+}\right)$, íon potássio $\left(\mathrm{K}^{+}\right)$e cálcio ionizado (Cai). Em todos os momentos de coleta sanguínea para 
hemogasometria, aferiu-se temperatura retal por meio de termômetro digital ultrarrápido (Termômetro digital Vicks ${ }^{\circledR}$ V911 ultrarrápido Kaz, Inc., Hudson, NY - USA).

Os resultados obtidos foram analisados com o programa SAS - Statistical Analysis System (SAS, 2005). Todos os parâmetros foram submetidos ao teste de normalidade e, em seguida, a análise entre os momentos foi realizada utilizando-se análise de variância (ANOVA) e teste de Tukey para comparação entre os tempos. Para todas as análises realizadas, foi adotado $\mathrm{P} \leq 0,05$ como nível de significância. Os dados foram apresentados como valores médios \pm desvio-padrão da média (DPM).

\section{RESULTADOS}

Dentre as variáveis hemogasométricas, os valores de $\mathrm{Na}, \mathrm{pH}, \mathrm{pO}_{2}, \mathrm{SO}_{2}, \mathrm{HCO}_{3}$ e $\mathrm{EB}$ não apresentaram diferença estatística entre os tempos. Os valores do íon $\mathrm{K}$, ao serem comparados com os valores basais, apresentaram diminuição significativa a partir de $\mathrm{T} 4(\mathrm{P} \leq 0,05)$, atingindo seu menor valor em T16. No entanto, após 36 horas de desobstrução (T40), os valores de íon $\mathrm{K}$ retornaram aos valores basais, mantendo-se até T76. O Cai apresentou os menores valores a partir de T4 em comparação aos valores basais $(\mathrm{P} \leq 0,01)$, retornando à normalidade após T40. Os valores da $\mathrm{pCO}_{2}$ apresentaram diferença estatística $(\mathrm{P} \leq 0,05)$, sendo a menor média obtida em T4 e a maior média em T1. A média e o desvio-padrão dos valores hemogasométricos encontram-se na Tab. 1. Os gráficos das variáveis que apresentaram diferença significativa encontramse representados nas Fig. 1 e 2.

Tabela 1. Valores da pressão parcial de oxigênio $\left(\mathrm{pO}_{2}\right)$, pressão parcial de dióxido de carbono $\left(\mathrm{pCO}_{2}\right)$, excesso/déficit de base (BE), saturação de oxigênio $\left(\mathrm{SO}_{2}\right)$, logaritmo negativo da atividade de íons hidrogênio $(\mathrm{pH})$, concentrações de bicarbonato $\left(\mathrm{HCO}_{3}{ }^{-}\right)$, íon sódio $\left(\mathrm{Na}^{+}\right)$, íon potássio $\left(\mathrm{K}^{+}\right)$e cálcio ionizado (Cai) dos equinos submetidos à obstrução experimental. Considera-se T0 antes do procedimento anestésico, T1 após a anestesia estabilizar, T4 o momento da desobstrução intestinal e T16, T28, T40, T52, T64 e T76 como pós-cirúrgico

\begin{tabular}{lccccccccc}
\hline & $\mathrm{T} 0$ & $\mathrm{~T} 1$ & $\mathrm{~T} 4$ & $\mathrm{~T} 16$ & $\mathrm{~T} 28$ & $\mathrm{~T} 40$ & $\mathrm{~T} 52$ & $\mathrm{~T} 64$ \\
$\mathrm{pO}_{2}$ & $35,12 \pm 3,22 \mathrm{a}$ & $44,25 \pm 27,81 \mathrm{a}$ & $31,12 \pm 6,19 \mathrm{a}$ & $34,87 \pm 4,32 \mathrm{a}$ & $36,33 \pm 3,55 \mathrm{a}$ & $35,0 \pm 4,43 \mathrm{a}$ & $33,16 \pm 4,07 \mathrm{a}$ & $32,42 \pm 3,59 \mathrm{a}$ & $34,83 \pm 3,65 \mathrm{a}$ \\
$\mathrm{pCO}_{2}$ & $45,20 \pm 2,41 \mathrm{bc}$ & $54,37 \pm 5,82 \mathrm{a}$ & $38,96 \pm 5,52 \mathrm{c}$ & $43,88 \pm 2,20 \mathrm{bc}$ & $43,16 \pm 1,33 \mathrm{bc}$ & $44,91 \pm 2,77 \mathrm{~b}$ & $45,11 \pm 2,27 \mathrm{~b}$ & $45,40 \pm 2,21 \mathrm{~b}$ & $46,35 \pm 3,25 \mathrm{~b}$ \\
$\mathrm{BE}$ & $4,87 \pm 2,53 \mathrm{ab}$ & $5,75 \pm 3,24 \mathrm{ab}$ & $8,25 \pm 3,37 \mathrm{a}$ & $3,12 \pm 3,68 \mathrm{~b}$ & $3,50 \pm 1,51 \mathrm{ab}$ & $4,28 \pm 2,49 \mathrm{ab}$ & $5,0 \pm 2,82 \mathrm{ab}$ & $4,42 \pm 3,95 \mathrm{ab}$ & $7,0 \pm 5,51 \mathrm{ab}$ \\
$\mathrm{SO}_{2}$ & $65,62 \pm 5,39 \mathrm{a}$ & $67,75 \pm 14,12 \mathrm{a}$ & $70,25 \pm 14,32 \mathrm{a}$ & $59,50 \pm 8,46 \mathrm{a}$ & $66,66 \pm 5,71 \mathrm{a}$ & $64,0 \pm 8,04 \mathrm{a}$ & $61,83 \pm 6,76 \mathrm{a}$ & $62,14 \pm 12,29 \mathrm{a}$ & $65,33 \pm 7,39 \mathrm{a}$ \\
$\mathrm{pH}$ & $7,418 \pm 0,02 \mathrm{a}$ & $7,365 \pm 0,05 \mathrm{a}$ & $7,458 \pm 0,20 \mathrm{a}$ & $7,409 \pm 0,03 \mathrm{a}$ & $7,418 \pm 0,02 \mathrm{a}$ & $7,416 \pm 0,01 \mathrm{a}$ & $7,420 \pm 0,01 \mathrm{a}$ & $7,416 \pm 0,03 \mathrm{a}$ & $7,432 \pm 0,04 \mathrm{a}$ \\
$\mathrm{HCO}_{3}{ }^{-}$ & $29,07 \pm 2,23 \mathrm{a}$ & $31,20 \pm 2,60 \mathrm{a}$ & $31,43 \pm 3,24 \mathrm{a}$ & $27,45 \pm 3,20 \mathrm{a}$ & $27,66 \pm 1,30 \mathrm{a}$ & $28,71 \pm 2,20 \mathrm{a}$ & $29,15 \pm 2,44 \mathrm{a}$ & $28,92 \pm 3,31 \mathrm{a}$ & $30,96 \pm 4,74 \mathrm{a}$ \\
$\mathrm{Na}^{+}$ & $137,37 \pm 1,99 \mathrm{a}$ & $138,0 \pm 2,07 \mathrm{a}$ & $138,0 \pm 2,87 \mathrm{a}$ & $137,75 \pm 2,91 \mathrm{a}$ & $137,33 \pm 2,87 \mathrm{a}$ & $136,85 \pm 1,95 \mathrm{a}$ & $135,50 \pm 3,93 \mathrm{a}$ & $134,0 \pm 4,32 \mathrm{a}$ & $131,16 \pm 13,61 \mathrm{a}$ \\
$\mathrm{K}^{+}$ & $4,23 \pm 0,32 \mathrm{a}$ & $3,73 \pm 0,45 \mathrm{abc}$ & $3,51 \pm 0,60 \mathrm{bcd}$ & $3,07 \pm 0,37 \mathrm{~d}$ & $3,11 \pm 0,58 \mathrm{~cd}$ & $3,94 \pm 0,27 \mathrm{ab}$ & $3,75 \pm 0,51 \mathrm{abc}$ & $3,82 \pm 0,48 \mathrm{ab}$ & $3,70 \pm 0,51 \mathrm{abcd}$ \\
$\mathrm{Cai}$ & $1,65 \pm 0,07 \mathrm{a}$ & $1,40 \pm 0,11 \mathrm{bc}$ & $1,24 \pm 0,10 \mathrm{c}$ & $1,49 \pm 0,12 \mathrm{ab}$ & $1,52 \pm 0,15 \mathrm{ab}$ & $1,66 \pm 0,08 \mathrm{a}$ & $1,65 \pm 0,11 \mathrm{a}$ & $1,61 \pm 0,13 \mathrm{a}$ & $1,65 \pm 0,25 \mathrm{a}$ \\
\hline
\end{tabular}

Médias seguidas pelas mesmas letras não diferem entre si pelo teste Tukey a $5 \%(\mathrm{P}<0,05)$.

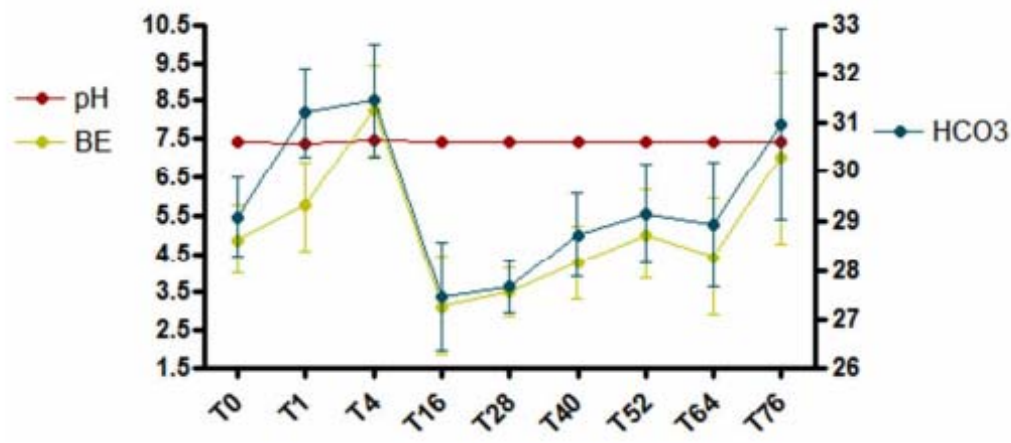

Figura 1. Valores do logaritmo negativo da atividade de íons hidrogênio ( $\mathrm{pH}$ ), concentrações de bicarbonato $\left(\mathrm{HCO}_{3}^{-}\right)$e excesso/déficit de base $(\mathrm{BE})$ aferidos de equinos submetidos à obstrução intraluminal. Considera-se T0 antes do procedimento anestésico, T1 após a anestesia estabilizar, T4 o momento da desobstrução intestinal e T16, T28, T40, T52, T64 e T76 como pós-cirúrgico. 


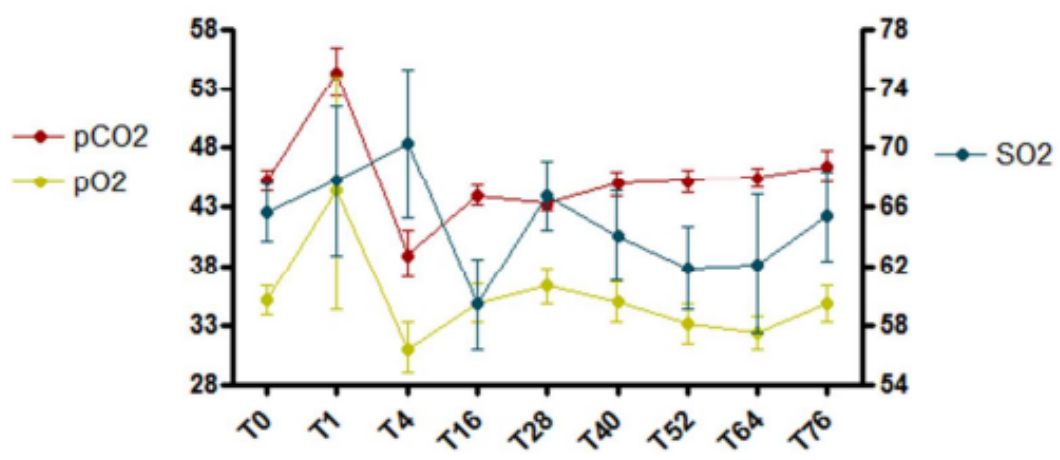

Figura 2. Valores da pressão parcial de oxigênio $\left(\mathrm{PO}_{2}\right)$, pressão parcial de dióxido de carbono $\left(\mathrm{PCO}_{2}\right)$ e saturação de oxigênio $\left(\mathrm{SO}_{2}\right)$ aferidos de equinos submetidos à obstrução intraluminal. Considera-se T0 antes do procedimento anestésico, T1 após a anestesia estabilizar, T4 o momento da desobstrução intestinal e T16, T28, T40, T52, T64 e T76 como pós-cirúrgico.

\section{DISCUSSÃO}

Mediante a avaliação hemogasométrica, foi possível observar que, após quatro horas do início da obstrução, ocorreu discreto aumento nos valores do $\mathrm{pH}$ associado com os aumentos de $\mathrm{HCO}_{3}{ }^{-}$e do $\mathrm{EB}$, o que confirma a ocorrência de alcalose metabólica inicial. Esse aumento transitório do $\mathrm{pH}$ é ocasionado pela perda de íons potássio do sangue. Quando as concentrações de potássio do líquido extracelular estão baixas, o potássio move-se do líquido intracelular para o extracelular e é substituído em parte por íons hidrogênio que são perdidos do plasma. A perda de íons hidrogênio libera tampão, de modo que a concentração plasmática de $\mathrm{HCO}_{3}^{-}$e a base tampão total aumentam, resultando na alcalose metabólica (Robinson, 2004).

Observou-se que, em T64 e T76, houve aumento da $\mathrm{pCO}_{2}, \quad$ caracterizando compensação respiratória. Segundo Johnson (1995), a acidose respiratória geralmente ocorre como mecanismo de compensação da alcalose metabólica ou como alteração primária em afecções pulmonares. A acidose respiratória, desencadeada pela hipoventilação, é responsável pela eliminação incompleta do dióxido de carbono pelos pulmões, provocando o aumento na $\mathrm{pCO}_{2}$ sanguínea. Consequentemente, ocorre acúmulo de íons $\mathrm{H}^{+}$, e, apesar do acúmulo simultâneo de bicarbonato, o $\mathrm{pH}$ diminui, pois a quantidade de bicarbonato acumulada é pequena para que ocorra a manutenção da proporção dos valores normais de 20:1 (Day, 2002).

Corroborando as afirmações anteriores e considerando que o $\mathrm{pH}$ normal de referência do sangue venoso de equinos encontra-se entre 7,32 e 7,44 (Carlson, 1997), constatou-se que as alterações apresentadas pelos animais deste estudo foram corrigidas por modificações respiratórias, o que difere dos resultados obtidos por Nappert e Johnson (2001) e por Di Filippo et al. (2008), os quais verificaram, em distúrbios abdominais de maior gravidade, que os animais não foram capazes de corrigir as alterações ácido-base. Provavelmente, a incapacidade desses casos deveu-se ao comprometimento da integridade e da perfusão renal, o que afetou a eliminação de íons $\mathrm{H}^{+}$e a reabsorção de bicarbonato nos rins (Di Filippo e Santana, 2007).

Nos animais deste estudo, as concentrações da $\mathrm{pO}_{2}$ e de $\mathrm{SO}_{2}$ apresentaram comportamento semelhante, diminuindo nos momentos T4, T16 e T28. Esses valores foram decorrentes do aumento da $\mathrm{pCO}_{2}$ ou hipoventilação, visando à correção da alcalose metabólica, mecanismo já descrito anteriormente. Resultados semelhantes foram obtidos por Ribeiro Filho et al. (2007) e por Di Filippo et al. (2008). A diminuição da $\mathrm{pO}_{2}$, observada por Ribeiro Filho et al. (2007), foi associada à ocorrência de um processo inflamatório pulmonar, detectado em três animais do estudo, nos quais a possível causa do processo inflamatório pulmonar foi o estresse presente nas fases de indução e de tratamento da compactação do cólon maior.

$\mathrm{O}$ EB é calculado com base no $\mathrm{pH}$ sanguíneo e na $\mathrm{pCO}_{2}$ e constitui importante parâmetro para identificar a ocorrência de acidose ou alcalose metabólica (Sucupira e Ortolani, 2003). 
Ocorreram alterações significativas nos valores médios de EB entre os tempos, e T4 apresentou maior valor. Essa variável se comportou de maneira semelhante ao $\mathrm{pH}$ sanguíneo, o que confirmou a ocorrência da alcalose metabólica nos animais deste estudo, já que seus valores permaneceram positivos até o final do período experimental. Acredita-se que tal elevação decorreu da somatória das alterações clínicopatológicas promovidas pela obstrução intraluminal no cólon menor.

A regulação do equilíbrio ácido-base é intimamente dependente do balanço de fluidos e eletrólitos, em que os rins possuem função significativa na manutenção da concentração fisiológica de vários eletrólitos plasmáticos. Dentre esses eletrólitos, destacam-se o sódio, o potássio e o cloreto (Rose, 1981). No presente estudo, constatou-se a diminuição nos valores do sódio nos animais, sendo apresentada como provável origem a perda de fluidos via gastrintestinal ou renal, como foi descrito por Rose (1981). Para Johnson (1995), a hiponatremia persistente ocorre apenas quando há grave comprometimento da função renal, pois deve-se considerar que quase todo sódio filtrado é absorvido no túbulo renal proximal. Os sinais clínicos relacionados com hiponatremia grave são manifestações neurológicas associadas ao edema cerebral, à letargia, aos tremores e às convulsões.

Ocorreu alteração quanto aos valores de potássio nos animais, assim como foi descrito por Fettman (2004). A hipocalemia pode estar associada à concentração de hormônios como a insulina e as catecolaminas. A excitação, a dor e o estresse, comuns nos quadros de cólica, desencadeiam a liberação de catecolaminas levando à hiperglicemia. A hiperglicemia instalada induz a hiperinsulinemia e a consequente hipocalemia. Ademais, como o potássio é absorvido por processo ativo, principalmente no intestino delgado, se existir quantidade elevada de sódio no intestino grosso, decorrente de processos patológicos, pode ocorrer absorção compensatória desse íon associada à secreção de potássio no cólon, resultando na perda de potássio nas fezes (Fettman 2004). Haskins (1988) também cita que a hipocalemia é comum em afecções intestinais agudas.
Segundo Garcia-Lopez et al. (2001), a determinação do cálcio pode ser útil na avaliação da gravidade da lesão, sendo a hipocalcemia um achado comum nos equinos com obstrução intraluminal. No presente estudo, o menor valor foi observado no momento T4, ou seja, logo no início da reperfusão. Esses resultados assemelham-se aos encontrados por GarciaLopez et al. (2011) em cães e seres humanos com doenças terminais. Trim (1990) citou que a hipocalcemia está relacionada à hipotensão e ao prejuízo à função cardiovascular. Múltiplos fatores, incluindo anorexia, endotoxemia e ressecção intestinal, podem estar associados à baixa concentração de cálcio em cães, condições essas comuns em equinos com o quadro de abdômen agudo. Os autores citam que as endotoxinas ou seus subprodutos podem causar hipocalcemia, durante a sepse, pelo prejuízo à mobilização desse cátion (Dart et al., 1992).

\section{CONCLUSÃO}

As alterações presentes na hemogasometria permitem a detecção de desordens metabólicas e podem auxiliar na elaboração do prognóstico e no acompanhamento da evolução do processo de cura dos animais acometidos pelo abdômen agudo em decorrência de leve obstrução simples.

\section{AGRADECIMENTOS}

Os autores agradecem à Fundação de Amparo à Pesquisa do Estado de São (Fapesp), pela ajuda financeira na realização deste projeto.

\section{REFERÊNCIAS}

ALVES, G.E.S.; RIBEIRO FILHO J.D.; OLIVEIRA H.P. et al. Tratamento da compactação experimental do cólon maior em equinos: resultados de laboratório e exames bioquímicos. Arq. Bras. Med. Vet. Zootec., v.57, p.281-287, 2005.

CARLSON, G.P. Fluid electrolyte, and acid-base balance. In: KANEKO, J.J.; HAENEY J.W.; BRUSS M.L. Clin. Biochem. Domest. Anim., v.5, 1997, p.485516.

CORRÊA, R.R.; ZOPPA, A.L.V.; SILVA, L.C.L.C. et al. Estudo retrospectivo dos casos de enterolitíase e corpo estranho em intestino grosso de equinos, no período de janeiro de 1993 a janeiro de 2003. Braz. J. Vet. Res. Anim. Sci., v.43, p.242-249, 2006. 
DART, A.J.; SNYDER, J.R.; SPIER, S.J. et al. Ionized calcium concentration in horses with surgically managed gastrointestinal disease: 147 cases (1988-1990). J. Am. Vet. Med. Assoc., v.201, p.12441248, 1992.

DAY, T.K. Blood gas analysis. Vet. Clin. N. Am. Small Anim. Pract., v.32, p.1031-1048, 2002.

DI FILIPPO, P.A.; SANTANA, A.E.; PEREIRA G.T. Equilíbrio ácido-base e hidroeletrolítico em equinos com cólica. Cienc. Rural, v.38, p.1003-1009, 2008.

DI FILIPPO, P.A.; SANTANA A.E. Variações nas concentrações dos biomarcadores sanguíneos da função renal e hepática em equinos com cólica. Vet. Notícias, v.13, p.47-54, 2007.

FALEIROS, R.R.; MACORIS, D.G.; ALVES, G.E.S. et al. Avaliação histomorfométrica e ultra-estrutural da mucosa do cólon menor equino submetido à distensão. Pesqui. Vet. Bras., v.27, p.383-387, 2007.

FETTMAN, M.J. Fluid and electrolyte metabolism. Vet. Hematol. Clin. Chem., v.22, p.329-355, 2004.

GARCIA-LOPEZ, J.M.; PROVOST, P.J.; RUSH, J.E. et al. Prevalence and prognostic importance of hypomagnesemia and hypocalcemia in horses that have colic surgery. Am. J. Vet. Res., v.62, p.7-12, 2001.

GOKCE, G.; CITIL, M.; GUNES, V. et al. Effect of the time delay and storage temperature on blood gas acid-base values of bovine venous blood. Res. Vet. Sci., v.76, p. 121-127, 2004.

GOSSET, K.A.; CLEGHORN, B.; ADAMS, R. et al. Contribution of whole blood L-lactate, pyruvate, D-lactate, acetoacetate, and 3-hydroxybutirate concentrations to the plasma anion gap in horses with intestinal disordens. Am. J. Vet. Res., v.48, p.72-75, 1987.

GRAVENA, K.; HERNANDEZ-TOVAR, M.C.; CANELLO, V.A. et al. Clinical and laboratorial changes in horses subjected to a high-pressure modified model of small colon distention. J. Equine Vet. Sci., v.42, p.32-38, 2016.

HASKINS, S.C. A simple fluid therapy planning guide. Semin. Vet. Med. Surg., v.3, p.227-236, 1988.

JOHNSON, P.J. Electrolyte and acid-base disturbances in the horse. Vet. Clin. N. Am. Equine Pract., v.11, p.491-514, 1995.
KLEIN, L.V.; SOMA, L.R.; NANN, L.E. Accuracy and precision of the portable StatPal II and the laboratory, based NOVA stat profile 1 for measurement oh $\mathrm{pH}, \mathrm{p}\left(\mathrm{CO}_{2}\right)$, and $\mathrm{p}\left(\mathrm{O}_{2}\right)$ in equine blood. Vet. Surg., v.28, p.67-76, 1999.

MARQUES, L.C. Equilíbrio hídrico-eletrolítico. Cólica Equina II: Diagnóstico e tratamento. In: CICLO DE PALESTRAS SOBRE CÓLICA EQUINA, 2., 1990, Jaboticabal. Anais... Jaboticabal: [s.n.], 1990. (Resumo).

NAPPERT G.; JOHNSON P. Determination of the acid-base status in 50 horses admitted with colic between December 1998 and May 1999. Can. Vet. J., v.42, p.703-707, 2001.

PEIRÓ, J.M.; MENDES, L.C. Semiologia do sistema digestório equino. In: FEITOSA, F.L.F. Semiologia veterinária: a arte do diagnóstico. São Paulo: Roca, 2004. p.139-175.

RIBEIRO FILHO, J.D.; ABREU, J.M.G.; ALVES, G.H.S. et al. Hemogasometria em equinos com compactação experimental do cólon maior tratados com sene, fluidoterapia enteral e parenteral. Cienc. Rural, v.37, p.755-761, 2007.

ROBINSON, N.E. Homeostase ácido básica. In: CUNNINGHAM, J.G. (Ed.). Tratado de fisiologia veterinária. Rio de Janeiro: Guanabara Koogan, v.51, 2004, p.539-550.

ROSE, R.J. A physiological approach to fluid and electrolyte therapy in the horse. Equine Vet. J., v.13, p.7-14, 1981.

SAS user's guide, version 9.1.3. Cary: SAS, 2005.

SILVERMAN, S.C.; BIRKS, E.K. Evaluation of the iSTAT hand-held chemical analyzer during treadmill and endurance exercise. Equine Vet. J., v.34, p.551$554,2002$.

SUCUPIRA M.C.A.; ORTOLANI E.L. Uso de sangue arterial e venoso no exame do equilíbrio ácido básico de novilhos normais ou com acidose metabólica. Cienc. Rural, v.33, p.863-868, 2003.

TRIM, C.M. Anesthesia for acute abdominal disease. In: WHITE, N.A. The equine acute abdomen. Philadelphia: Lea \& Febiger, 1990. v.1, p.189-207. 